\title{
H3F3A mutant allele specific imbalance in an aggressive subtype of diffuse midline glioma, H3 K27M-mutant
}

\author{
Sachi Maeda', Fumiharu Ohka ${ }^{\text {* }}$, Yusuke Okuno², Kosuke Aoki ${ }^{1}$, Kazuya Motomura ${ }^{1}$, Kazuhito Takeuchi ${ }^{1}$, \\ Hironao Kusakari ${ }^{3}$, Nobuyuki Yanagisawa ${ }^{3}$, Shinya Sato ${ }^{4}$, Junya Yamaguchi ${ }^{1}$, Kuniaki Tanahashi ${ }^{1}$, Masaki Hirano ${ }^{1}$, \\ Akira Kato ${ }^{1}$, Hiroyuki Shimizu ${ }^{1}$, Yotaro Kitano ${ }^{1}$, Shintaro Yamazaki ${ }^{1}$, Shinji Yamashita ${ }^{5}$, Hideo Takeshima ${ }^{5}$, \\ Keiko Shinjo ${ }^{6}$, Yutaka Kondo ${ }^{6}$, Toshihiko Wakabayashi ${ }^{1}$ and Atsushi Natsume ${ }^{{ }^{*}}$
}

\begin{abstract}
Diffuse midline glioma, H3 K27M-mutant is a lethal brain tumor located in the thalamus, brain stem, or spinal cord. H3 K27M encoded by the mutation of a histone H3 gene such as H3F3A plays a pivotal role in the tumorigenesis of this type of glioma. Although several studies have revealed comprehensive genetic and epigenetic profiling, the prognostic factors of these tumors have not been identified to date. In various cancers, oncogenic driver genes have been found to exhibit characteristic copy number alterations termed mutant allele specific imbalance (MASI). Here, we showed that several diffuse midline glioma, H3 K27M-mutant exhibited high variant allele frequency (VAF) of the mutated H3F3A gene using droplet digital polymerase chain reaction (ddPCR) assays. Whole-genome sequencing (WGS) revealed that these cases had various copy number alterations that affected the mutant and/or wild-type alleles of the H3F3A gene. We also found that these MASI cases showed a significantly higher Ki-67 index and poorer survival compared with those in the lower VAF cases $(P<0.05)$. Our results indicated that the MASI of the H3F3A K27M mutation was associated with the aggressive phenotype of the diffuse midline glioma, H3 K27Mmutant via upregulation of the $\mathrm{H} 3 \mathrm{~K} 27 \mathrm{M}$ mutant protein, resulting in downregulation of H3K27me3 modification.
\end{abstract}

Keywords: Diffuse midline glioma, H3 K27M-mutant, Mutant allele specific imbalance, Whole-genome sequencing, Droplet digital polymerase chain reaction

\section{Introduction}

Diffuse midline glioma is an infiltrative glial neoplasm located in the thalamus, brain stem, or spinal cord. Most of these tumors develop in children, adolescents, and young adults with a lethal clinical course. Most cases cannot achieve maximum tumor removal owing to tumor location and effective adjuvant therapies other than radiation therapy have not been identified to date. Recent comprehensive molecular analyses revealed that the Lys 27-to-methionine (K27M) mutations at one allele of the histone $\mathrm{H} 3$ gene such as the $H 3 F 3 A$ gene were found in approximately $80 \%$ of diffuse intrinsic pontine gliomas (DIPGs), 50\% of thalamic tumors, and

\footnotetext{
*Correspondence: fohka@med.nagoya-u.ac.jp; anatsume@med.nagoya-u.ac.jp ${ }^{1}$ Department of Neurosurgery, Nagoya University Graduate School of Medicine, 65 Tsurumai-cho, Showa-ku, Nagoya 466-8550, Japan Full list of author information is available at the end of the article
}

$60 \%$ of spinal tumors [1-6]. Diffuse midline glioma exhibiting heterozygous $\mathrm{H} 3 \mathrm{~K} 27 \mathrm{M}$ mutation is defined as diffuse midline glioma, H3 K27M-mutant by the 2016 World Health Organization Classification of Tumors of the Central Nervous System [7]. H3 K27M protein encoded by the H3 K27M mutated allele of this gene plays a pivotal role in tumor formation via the global loss of the H3K27me3 level [3, 8-10]. Molecular mechanisms during diffuse midline glioma, H3 K27M-mutant formation have been well-studied; however, the prognostic markers of this type of glioma have not been identified to date.

Several studies have reported that various cancers exhibit mutant allele specific imbalance (MASI) of driver oncogenes [11-16]. The copy number gain of the mutant allele and/or loss of the wild-type allele of these genes constitutes MASI. In KRAS-mutant pancreatic

(c) The Author(s). 2020 Open Access This article is distributed under the terms of the Creative Commons Attribution 4.0 International License (http://creativecommons.org/licenses/by/4.0/), which permits unrestricted use, distribution, and 
adenocarcinomas and colorectal cancers, MASI of the $K R A S$ gene has been associated with a poorer prognosis compared with tumors with heterozygous KRAS mutations [17]. The MASI of the driver oncogene might be a new class of potential biomarkers for malignancy, even though the MASI of the H3F3A gene in the diffuse midline glioma, H3 K27M-mutant has not been identified yet.

In the present study, we found that a subset of the diffuse midline glioma, H3 K27M-mutant exhibited high variant allele frequency (VAF) of H3F3A K27M mutation using droplet digital polymerase chain reaction (ddPCR). Whole-genome sequencing (WGS) revealed the MASI of the H3F3A K27M mutation in these cases. We found that MASI cases exhibited a significantly higher Ki-67 index and poorer survival together with a lower H3K27me3 level compared with lower VAF cases. These data suggest that the MASI of H3F3A K27M mutation contributed to an aggressive phenotype of the diffuse midline glioma, H3 K27M-mutant.

\section{Materials and methods \\ DNA extraction from tumor and blood samples}

Tumor samples were obtained intra-operatively. DNA was extracted from frozen tumor and blood samples using a QIAamp DNA Mini Kit (Qiagen, Hilden, Germany) according to the manufacturer's instructions. The amount of obtained DNA was evaluated by the Qubit dsDNA HS Assay Kit (Invitrogen, Paisley, Scotland). We also extracted DNA from formalin-fixed paraffin-embedded (FFPE) tumor samples using the GeneRead DNA FFPE Kit (Qiagen) according to the manufacturer's instructions.

\section{Sanger sequencing}

We performed Sanger sequencing for the detection of H3F3A gene mutations in DNA extracted from the tumor samples. We amplified a 194-base pair (bp) fragment for DNA spanning the sequence encoding histone H3 Lysine (K) 27 of the H3F3A gene. We applied a conventional PCR involving the following steps: 35 cycles with denaturation at $98^{\circ} \mathrm{C}$ for $10 \mathrm{~s}$, annealing at $55^{\circ} \mathrm{C}$ for $30 \mathrm{~s}$, and extension at $68^{\circ} \mathrm{C}$ for $30 \mathrm{~s}$, with a final extension step at $68^{\circ} \mathrm{C}$ for $5 \mathrm{~min}$; the forward primer $\left(5^{\prime}\right.$-TGCTGGTAGG TAAGTAAGGAG-3') and reverse primer ( $5^{\prime}$-AGCAGT AGTTAAGTGTTCAAATG-3') were used. Sequence analysis was performed by ApE v2.0.55.

\section{Droplet digital PCR (ddPCR)}

To evaluate the VAF of the $H 3 F 3 A K 27 M$ mutation, we performed ddPCR on all samples. The reaction mixture for ddPCR comprised $10 \mu \mathrm{l}$ of $2 \times \mathrm{ddPCR}$ Supermix for probes (UniqueAssayID: dHsaMDV2510510; Bio-Rad Laboratories, Pleasanton, CA, USA), and $1 \mu \mathrm{l}$ of $20 \times$ target (FAM) and wild-type (HEX) probe with $30 \mathrm{ng}$ of DNA from each tumor specimen. Each mixture was mixed with $65 \mu \mathrm{l}$ of Droplet Generation Oil (Bio-Rad Laboratories) and droplets were generated using the QX200 Droplet Generator (Bio-Rad Laboratories). PCR amplification was performed on a $\mathrm{C} 1000$ thermo cycler (Bio-Rad Laboratories) with the following thermal cycling conditions: 40 cycles of denaturation of $94{ }^{\circ} \mathrm{C}$ for 30 $\mathrm{s}$, annealing and extension of $55^{\circ} \mathrm{C}$ for $60 \mathrm{~s}$ with a ramp rate of $2{ }^{\circ} \mathrm{C} / \mathrm{s}$. Next, the fluorescence intensity of each droplet was calculated using a QX200 Droplet Reader (Bio-Rad Laboratories) and analyzed using QuantaSoft droplet reader software (Bio-Rad Laboratories). VAF was calculated by the number of positive dots of FAM probes/(those of FAM + those of HEX).

\section{Construction of H3F3A wild-type and K27M mutant plasmid}

To validate VAF values measured by ddPCR, we constructed a pcDNA3.0 plasmid containing each wild-type $H 3 F 3 A$ and $H 3 F 3 A K 27 M$. The construct of wild-type $H 3 F 3 A$ and $H 3 F 3 A$ K27M sequences were obtained by PCR amplification using the DNA of each case. The forward primer (5'-CGAAGCTTAGGCCGTTCGAGGT AATTTTT-3') and reverse primer (5'-ATGGATCCGG TCTCCTTAGACCTCCAGGTAA-3') were used. The amplified PCR product was digested with $\mathrm{BamHI}$ and HindIII and ligated into a multi-cloning site of pcDNA3.0 vector using the Ligation Mix (Takara bio, Shiga, Japan).

\section{Immunohistochemistry (IHC)}

FFPE tissue was sectioned and deparaffinized and antigen retrieval was performed by microwave oven heating in sodium citrate buffer $(10 \mathrm{mM}, \mathrm{pH} 6.0)$ at $95^{\circ} \mathrm{C}$ for 20 min. To block endogenous peroxidase, slides were dipped in $3 \% \mathrm{H}_{2} \mathrm{O}_{2}$ with methanol for $10 \mathrm{~min}$ at room temperature. The slides were subsequently blocked in Dako Antibody Diluent (Dako, Glostrup, Denmark) for $10 \mathrm{~min}$ at room temperature. The primary antibodies were diluted in blocking buffer and incubated overnight at $4{ }^{\circ} \mathrm{C}$. We used rabbit monoclonal anti-histone $\mathrm{H} 3$ K27M antibody (RevMAb Biosciences, San Francisco, CA, USA), mouse monoclonal anti-histone $\mathrm{H} 4$ antibody (Cell signaling, Beverly, MA), rabbit monoclonal antitrimethyl-histone H3 (Lys27) antibody (Merck Millipore, Darmstadt, Germany), and mouse anti-Ki-67 antigen antibody (Dako). Secondary antibodies were goat antirabbit IgG $(\mathrm{H}+\mathrm{L})$ highly cross-absorbed secondary antibody, Alexa fluor 546 (Thermo Fisher Scientific, Waltham, MA, USA), or goat anti-mouse $\operatorname{IgG}(\mathrm{H}+\mathrm{L})$ highly cross-absorbed secondary antibody, Alexa fluor 488 (Thermo Fisher Scientific). Slides were stained with DAPI using VECTASHIELD mounting medium with 
DAPI (Vector Laboratories, Burlingame, CA). A BZX710 microscope (Keyence, Osaka, Japan) was used for fluorescence microscopy.

\section{Image analysis of IHC staining of tumor sections}

For image analyses upon IHC staining of the samples, all the images were obtained using appropriate conditions and thus, consistent findings were obtained for various control samples. The tumor content of the tumor specimens was calculated as $\mathrm{H} 3 \mathrm{~K} 27 \mathrm{M}$ positive cell numbers/ DAPI positive cell numbers. The frequency of $\mathrm{Ki}-67$ positive cells in tumor cells was calculated as Ki-67 positive cell numbers/H3 K27M positive cell numbers. The frequency of H3K27me3 positive cells was calculated as the H3K27me3 positive cell numbers/histone $\mathrm{H} 4$ positive cell numbers. The fluorescent intensity of H3 K27M and histone $\mathrm{H} 4$ was quantified by converting intensity to a gray scale based on the RGB color model using Adobe Photoshop CS6 (Adobe Systems Incorporated, San Jose, $\mathrm{CA}$ ). The relative fluorescence intensity of H3 K27M was calculated as the florescence intensity of H3 K27M/ florescence intensity of histone $\mathrm{H} 4$ in each tumor cell. Median or mean values of these evaluated values were calculated among those obtained in four fields.

\section{Whole-genome sequencing (WGS)}

DNA libraries were prepared using TruSeq DNA PCRFree Library Prep Kit (Illumina, San Diego, CA, USA) according to the manufacturer's instructions. The NovaSeq6000 platform (Illumina) was used to sequence and generate 400-bp long paired-end reads. A median of $717,605,970$ reads per sample was obtained and aligned to cover the hg19 reference genome with $33.2 x$ coverage using the Burrows-Wheeler aligner (http:// bio-bwa.sourceforge.net/) with default parameters and a -mem option. Sequence variations were detected and annotated using VarScan2 (http://dkoboldt.github.io/ varscan/). For copy number analysis, the coverage of each $10 \mathrm{~kb}$ span was compared with the mean coverage of the entire genome. The data was segmented by circular binary segmentation using DNAcopy 1.56 .0 with the modified parameters [18]. We also evaluated the mismatch ratio of the common single nucleotide polymorphisms (SNPs), which were reported to be found with more than $5 \%$ frequency. The moving average of three sequential median values of mismatch ratio of SNPs located on each $10 \mathrm{~kb}$ region was calculated. B allele frequency (BAF) was calculated by the moving average of mismatch ratio and (1-moving average of mismatch ratio) in each SNP region. Higher or lower values between the moving average of the mismatch ratio and (1-moving average of mismatch ratio) were described as yellow or green dots, respectively.

\section{Targeted-capture sequencing}

Sequencing libraries were prepared according to the manufacturer's protocol using a SureSelect XT target enrichment system and ClearSeq SS Comprehensive Cancer bait that targeted 151 cancer-associated genes (Agilent technologies, Santa Clara, CA). Prepared libraries were run on a Miseq next-generation sequencing platform using Miseq Reagent kit v3 (Illumina). The sequenced reads were aligned to the hg19 reference genome using the Burrows Wheeler aligner with default parameters and a -mem option. PCR duplicates were removed using Picard tools (http://broadinstitute.github. io/picard/). Sequence variations were detected and annotated using VarScan 2 and the variants that fulfilled the following criteria were adopted for further analyses: (i) number of variant reads $>10$, and (ii) variants allele frequency $\geq 0.05$. The adopted variants were annotated with ANNOVAR (http://annovar.openbioinformatics.org/en/ latest/) and the variants that met at least one of the following criteria were excluded from the analyses: (i) synonymous and ambiguously annotated (unknown) variants, (ii) common SNPs which were defined as those with $\geq 1 \%$ allele frequency in the Exome Aggregation Consortium (ExAC) (http://exac.broadinstitute.org/), the 1000 Genomes Project (http://www.internationalgenome.org/), and National Heart, Lung, and Blood Institute (NHLBI) Exome Sequencing Project (ESP) 6500 (https:// evs.gs.washington.edu/EVS/) or registered in NCBI dbSNP build 138 (https://www.ncbi.nlm.nih.gov/snp/).

\section{Fluorescence in situ hybridization (FISH)}

FISH assay was performed according to the manufacturer's instructions with minor modifications. Briefly, the FFPE tissue sections were deparaffinized with xylene. These samples were then pretreated using pretreatment buffer supplied in the Vysis paraffin Pretreatment IV \& Post-hybridization Wash Buffer kit (Abbott laboratories, Chicago, IL). Aquarius Pathology Probes 1p36/1q25 (Riken genesis CO., LTD. Tokyo, Japan) were applied to the pretreated sections. Samples and probes were denatured at $80^{\circ} \mathrm{C}$ for $3 \mathrm{~min}$ prior to the application, followed by a hybridization step at $37^{\circ} \mathrm{C}$ for $48 \mathrm{~h}$. Then, the sections were subjected to the washing step using wash buffer supplied in the Vysis paraffin Pretreatment IV \& Post-hybridization Wash Buffer kit (Abbott laboratories). The sections were then stained with DAPI, mounted on a slide with mounting medium (Riken genesis CO., TLD.) and were further assessed under a Nikon fluorescent microscope with appropriate filters (Nikon instruments, Tokyo, Japan).

\section{Western blot analysis}

Cell lysate was extracted from FFPE tissue samples using a Qproteome FFPE Tissue Kit (Qiagen). $15 \mu \mathrm{g}$ of total 
protein from each sample was run on $4-12 \%$ bis-tris precast polyacrylamide gel (Thermo Fisher Scientific), transferred to PVDF membrane using a wet system and probed overnight with primary antibody. The following primary antibodies were used: rabbit polyclonal antiHistone H3 antibody (Abcam, Cambridge, MA), rabbit monoclonal anti-histone H3 K27M (RevMAb Biosciences) and rabbit monoclonal anti-trimethyl-histone $\mathrm{H} 3$ (Lys27) antibody (Merck Millipore). The blot was then probed with the rabbit HRP-conjugated secondary antibodies (GE Healthcare, Buckinghamshire, UK). Membranes were revealed using chemiluminescent detection reagent (GE Healthcare) and enhanced signal was detected in CCD imaging system. Protein extracted from the FFPE tumor sample of $H 3 F 3 A$ wild-type glioma (IDH1 mutated) case was used as a negative control for $\mathrm{H} 3 \mathrm{~K} 27 \mathrm{M}$ and as a positive control for H3K27me3 [19].

\section{Statistical analysis}

The statistical significance of differences between two groups was analyzed by paired Student t-test, Wilcoxon signed-rank test, and Wilcoxon rank-sum test with $\mathrm{R}$ Statistical Software (version 3.6.1; R Foundation for Statistical Computing, Vienna, Austria). Overall survival (OS) was calculated as the time of tumor removal until death or last follow-up. Progression-free survival (PFS) was calculated as the time of tumor removal until recurrence or last follow-up. OS and PFS were evaluated using a log-rank test and Cox proportional hazards regression modeling. All reported $P$ values were two-sided, with $P<0.05$ considered statistically significant.

\section{Results}

\section{DdPCR analyses of H3F3A K27M mutation}

DdPCR calculated the VAF of the H3F3A K27M mutated genes. To analyze the correlation between the calculated VAF with ddPCR and the expected VAF, we performed ddPCR with a serial proportional mixture of plasmid DNAs containing the H3F3A K27M mutated sequence and those of the $H 3 F 3 A$ wild-type (Fig. 1a). We detected a significant correlation between the calculated VAF and expected VAF $\left(P<0.01 ; r^{2}=0.99\right)$ (Fig. 1b). Using this assay, we analyzed 15 cases of diffuse midline glioma, H3 K27M-mutant (Table 1). Four out of 15 diffuse midline glioma, H3 K27M-mutant cases exhibited more than 50\% VAF (Fig. 1c and Table 1). Sanger sequencing also revealed a higher mutant peak than that of the wild-type allele in these four cases (Fig. 1d). DdPCR revealed that three (case 10, 12 and 15) out of the four cases whose blood samples were available exhibited no germline $H 3 F 3 A K 27 M$ mutations. These data indicated that the four cases might exhibit MASI of H3F3A K27M mutation. Therefore, we performed WGS using DNA derived from the four cases that exhibited more than $50 \%$ VAF.

\section{MASI of H3F3A K27M mutation identified by WGS}

In the four MASI cases included in the present study, we analyzed the copy number of all the chromosomes meticulously using WGS (Additional file 1: Figure S1). For chromosome 1, where the $H 3 F 3 A$ gene is located, we also analyzed the BAF of the common SNPs. We evaluated the tumor content of the tumor specimen as the frequency of $\mathrm{H} 3 \mathrm{~K} 27 \mathrm{M}$ positive cells among DAPI positive cells. By combining these data with the VAF of the $H 3 F 3 A K 27 M$ obtained by ddPCR, we determined the most appropriate chromosomal structure model for each case. Based on the total copy numbers of chromosome 1 obtained by WGS, we determined two candidate chromosome 1 copy number models exhibiting (i): copy number of integral value which is the closest to the mean value of copy number obtained by WGS and (ii): (i) +1 in cases where copy number obtained by WGS was more than two, or (i) - 1 in cases where copy number obtained by WGS was less than two. This is because normal cells (copy number: two) which were included in tumor tissue samples might decrease the calculated copy number value of tumor cells whose copy numbers were more than two, or increase that of tumor cells whose copy numbers were less than two. Next, in each candidate copy number model, we calculated the expected BAFs of the common SNPs on chromosome 1 using the tumor content of the tumor specimen. In these models, we selected two copy number models exhibiting the most consistent BAFs with those obtained by WGS. Next, we calculated the expected VAF of the $H 3 F 3 A$ $K 27 M$ in each selected candidate model using the tumor content of the tumor specimen. Then, we identified the most appropriate chromosomal structure model exhibiting more consistent expected VAF with that obtained by ddPCR in the two candidate models. Finally, we calculated the expected tumor content using the VAF of the $H 3 F 3 A K 27 M$ obtained by ddPCR in this model and compared the expected tumor content with that of the tumor specimen for validation (Fig. 2). For cases 5 and 15, WGS revealed candidate models that exhibited an one copy loss of $1 \mathrm{p}$ and one or two copy gains of $1 \mathrm{q}$ in tumor cells (Fig. 3a). For case 5, we calculated the expected BAF of SNPs using the tumor content of the tumor specimen (64.2\%; Fig. 3b). Then, we selected two models that exhibited the most consistent expected BAF of SNPs (86.4 and 89.1\%) with that obtained by WGS (91.0\%; Fig. 3a). Next, we calculated the expected VAF of the H3F3A K27M in these models (72.9 and 78.2\%) using the tumor content of the tumor specimen $(64.2 \%$; Fig. 3b). We selected the model exhibiting the more consistent expected VAF of H3F3A K27M (72.9\%) with that 
a

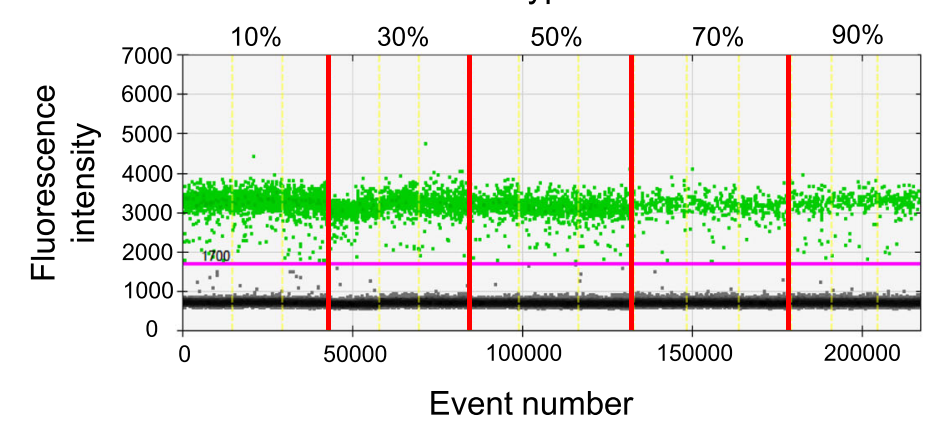

FAM: H3F3A K27M

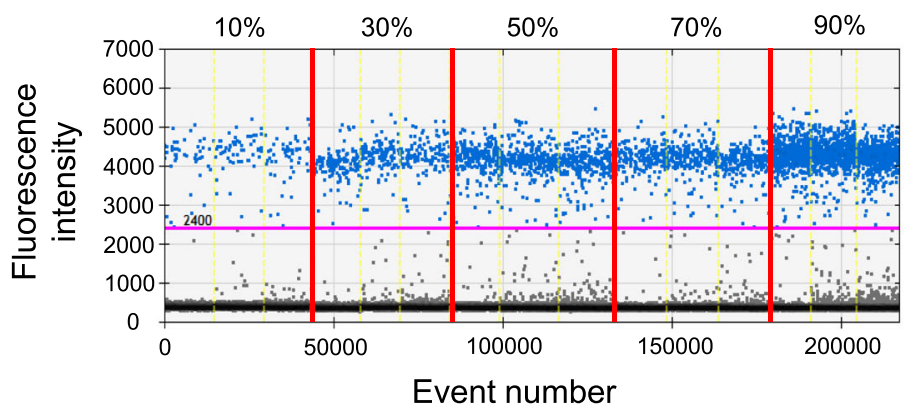

b

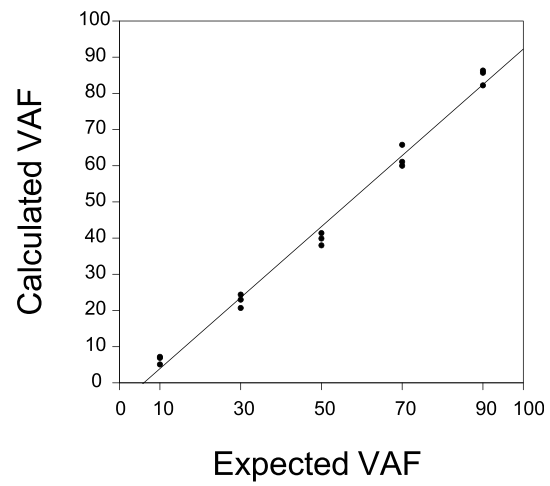

C

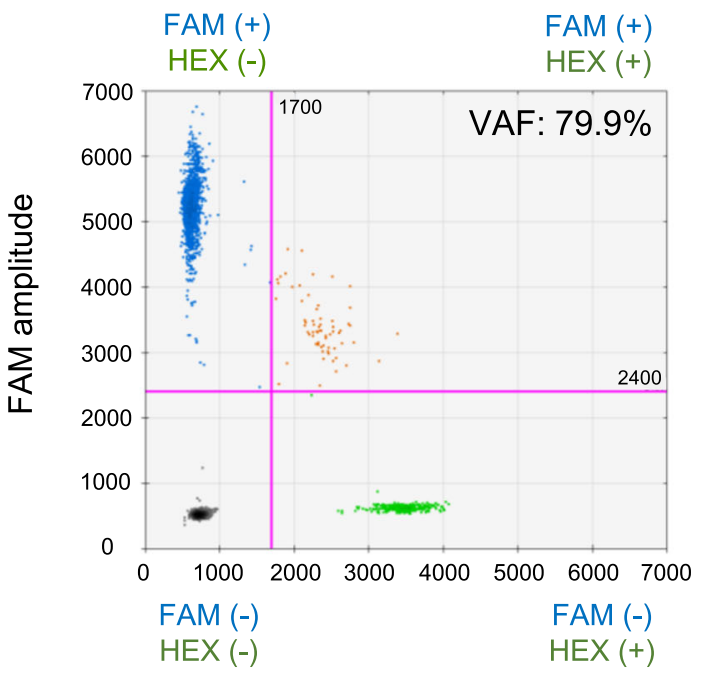

d

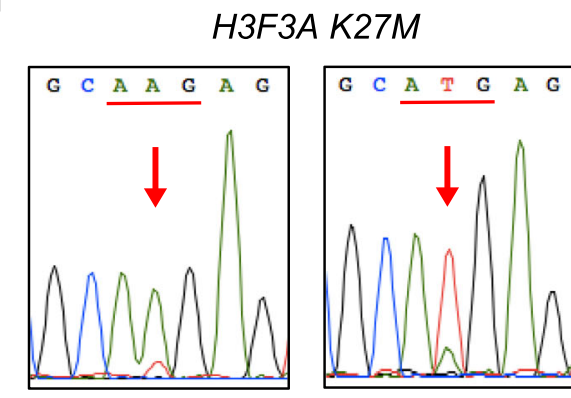

Lower VAF case

(VAF: 13.1\%)

\section{MASI case \\ (VAF: $79.9 \%$ )}

\section{HEX amplitude}

Fig. 1 Genetic analyses of H3F3A gene mutation using ddPCR and Sanger sequencing. a Results of ddPCR using various mixture DNA consisting of H3F3A K27M and wild-type H3F3A. Upper dot plots indicate wild-type H3F3A DNA dots (green) and lower dot plots indicate H3F3A K27M DNA dots (blue). The $x$-axis is the number of droplets including PCR products. The $y$-axis shows the intensity of HEX and FAM. The pink line shows the threshold (HEX: 1700, FAM: 2400) b Correlation of VAFs calculated by ddPCR with expected VAF. The $x$-axis is the expected VAF and the $y$-axis is VAF calculated by ddPCR $\left(P<0.01, r^{2}=0.99\right)$. c 2 D cluster plot of droplet fluorescence of H3F3A K27M and wild-type H3F3A dots in more than $50 \%$ VAF of H3F3A K27M case (case 12). FAM positive and HEX negative droplets (blue) include H3F3A K27M. HEX positive and FAM negative droplets (green) include wild-type H3F3A. VAF of H3F3A K27M was calculated as (blue dot numbers/blue dot numbers + green dot numbers). d Sanger sequencing of lower than 50\% VAF case (left; $13.1 \%$ of VAF; case 8 ) or more than 50\% VAF case (right; $79.9 \%$ of VAF; case 12 ). Hot spots of H3F3A K27M mutation are indicated by the red arrows 
Table 1 Summary of clinical features, VAF of H3F3A K27M and relative H3 K27M fluorescence intensity

\begin{tabular}{|c|c|c|c|c|c|}
\hline Case Number & Age at onset & Sex & Tumor location & $\begin{array}{l}\text { VAF of } \\
\text { H3F3A K27M (\%) }\end{array}$ & $\begin{array}{l}\text { Relative } \mathrm{H} 3 \mathrm{~K} 27 \mathrm{M} \text { fluorescence } \\
\text { intensity(Median) }\end{array}$ \\
\hline 1 & 6 & $\mathrm{~F}$ & Pons & 40.7 & ${ }^{\mathrm{a}} \mathrm{N} / \mathrm{A}$ \\
\hline 2 & 30 & $\mathrm{~F}$ & Insular gyrus & 42.4 & 1.13 \\
\hline 3 & 53 & M & Brainstem, Temporal lobe & 0.8 & 0.23 \\
\hline 4 & 14 & M & Spinal Cord & 38.3 & 1.27 \\
\hline 5 & 37 & M & Brainstem & 73.2 & 1.80 \\
\hline 6 & 37 & $\mathrm{~F}$ & Thalamus, Ventricle, Midbrain & 40.7 & 1.07 \\
\hline 7 & 15 & $\mathrm{~F}$ & Basal ganglia & 29.6 & ${ }^{\mathrm{a}} \mathrm{N} / \mathrm{A}$ \\
\hline 8 & 29 & M & Thalamus & 13.1 & 0.52 \\
\hline 9 & 31 & $\mathrm{~F}$ & Thalamus & 42.8 & ${ }^{\mathrm{a}} \mathrm{N} / \mathrm{A}$ \\
\hline 10 & 14 & M & Spinal Cord & 68.0 & 1.98 \\
\hline 11 & 9 & $\mathrm{~F}$ & Brainstem & 22.7 & 0.81 \\
\hline 12 & 31 & $\mathrm{~F}$ & Brainstem, Thalamus, Cerebellum & 79.9 & 1.50 \\
\hline 13 & 14 & M & Thalamus & 49.6 & ${ }^{\mathrm{a}} \mathrm{N} / \mathrm{A}$ \\
\hline 14 & 24 & M & Corpus callosum & 43.0 & ${ }^{\mathrm{a}} \mathrm{N} / \mathrm{A}$ \\
\hline 15 & 21 & M & Corpus callosum & 65.9 & 1.09 \\
\hline
\end{tabular}

aN/A not available

obtained by ddPCR (73.2\%). We found that the chromosomal structure model that exhibited three H3F3A $K 27 M$ mutant alleles without the wild-type allele was the most appropriate (Fig. 3c and Additional file 2: Figure S2). For case 15, we selected the two models that exhibited the most consistent BAF of SNPs (66.5 and $74.8 \%$ ) calculated using the tumor content of the tumor specimen (98.8\%; Fig. 3b) with that obtained by WGS (66.0\%; Fig. 3a). We selected the chromosomal structure model that exhibited the more consistent VAF of the H3F3A K27M mutant (66.1\%) calculated using the tumor content of the tumor specimen (98.8\%; Fig. 3b) with that obtained by ddPCR $(65.9 \%)$. We found that the chromosomal structure model exhibiting two H3F3A $K 27 M$ mutant alleles with one wild-type allele (a total of three copies) was the most appropriate (Fig. 3c and Additional file 3: Figure S3). For case 10, the WGS indicated two candidate models that exhibited two or three

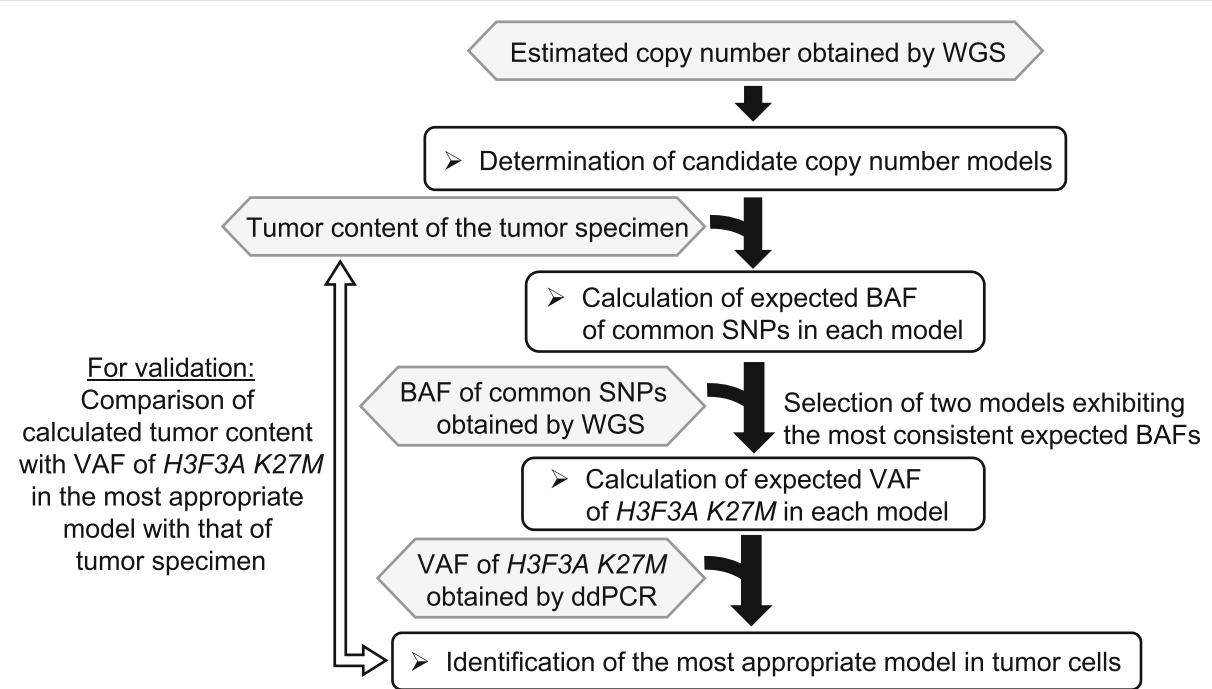

Fig. 2 Flowchart for the identification of the most appropriate chromosomal structure model. Using the total copy number of chromosome 1 obtained by WGS, tumor content of the tumor specimen (H3 K27M positive cells/DAPI positive cells in IHC), BAF of common SNPs obtained by WGS, and VAF of H3F3A K27M obtained by ddPCR, we identified the most consistent chromosomal structure model. Then, we calculated the tumor content with VAF of H3F3A K27M in the most appropriate model and compared the calculated tumor content with that of the tumor specimen obtained by $\mathrm{IHC}$ for validation 


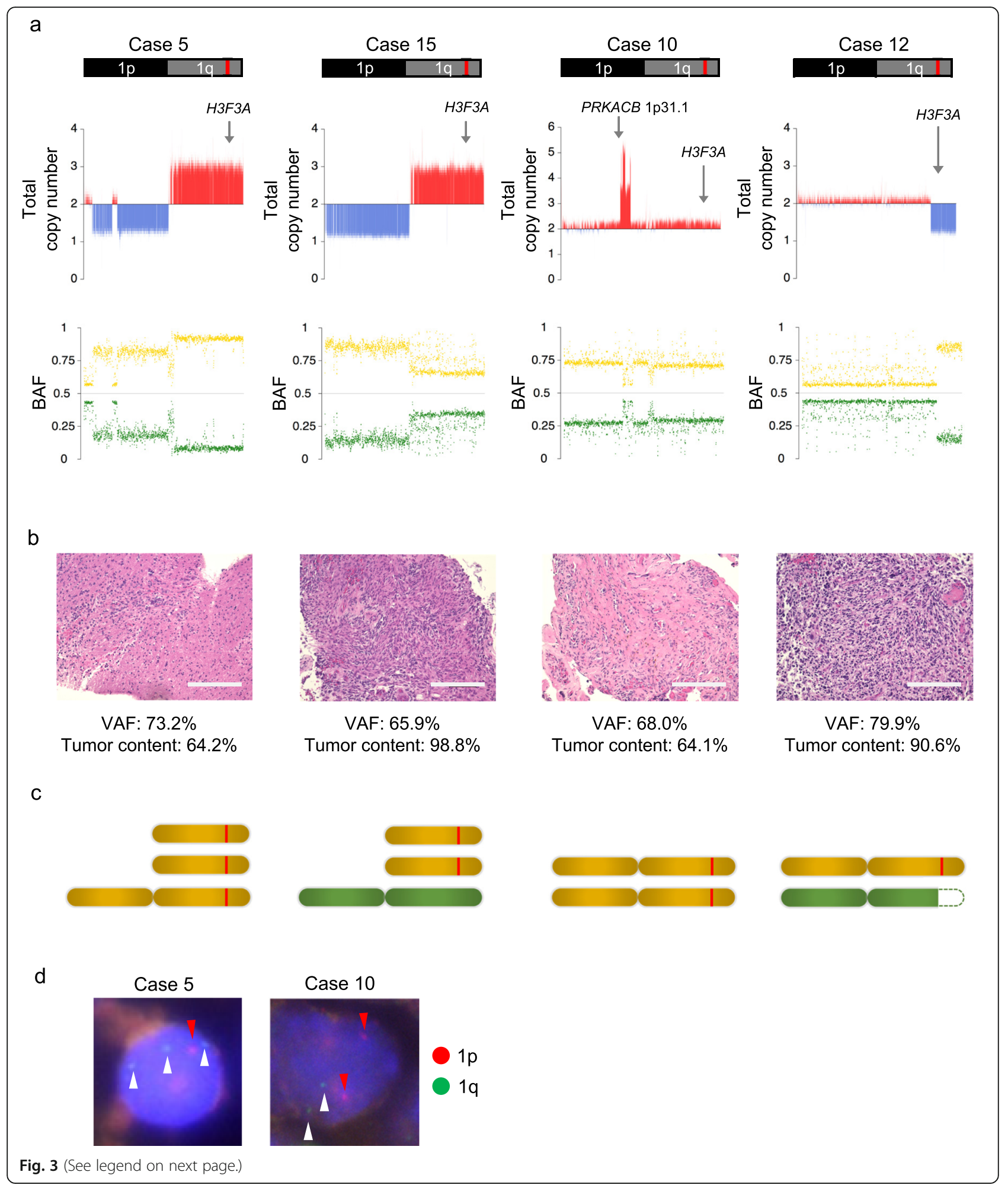


(See figure on previous page.)

Fig. 3 MASI of H3F3A K27M mutation determined with whole-genome sequencing. a Total copy number analyses of chromosome 1 on which H3F3A gene located (upper) and B allele frequency (BAF) of common SNPs (lower) with whole-genome sequencing (WGS) of four more than 50\% VAF cases (cases 5, 15, 10, and 12). The red line shows the H3F3A gene region. The $x$-axis shows the gene location and the $y$-axis shows the copy number. Gray arrows indicate the H3F3A gene region and PRKACB gene region. Red and blue bars indicate gain and loss, respectively (upper). Higher or lower values between the moving average of the mismatch ratio of SNPs and (1-moving average of mismatch ratio of SNPS) are shown as a yellow or green dot, respectively (lower). b Hematoxylin-Eosin staining of tumor specimen (case 5, 15, 10, and 12; bars: 200um). In each case, the tumor content was calculated as H3 K27M positive cell numbers/DAPI positive cell numbers. c Expected chromosomal structure models of chromosome 1 in cases 5, 15, 10, and 12. The red line indicates the H3F3A K27M mutation. $\mathbf{d}$ FISH analysis of chromosome 1p and 1q in MASI cases (case 5; left and case 10; right). Red arrow heads indicated 1 p36 signals (red) and white arrow heads indicated 1q25 signals (green) in DAPI-stained nuclei

copies of the whole chromosome 1 with focal amplification including the PRKACB gene, whose amplification in pediatric high-grade glioma has been reported previously [20] (Fig. 3a). We calculated the expected BAF of SNPs using the tumor content of the tumor specimen $(64.1 \%$; Fig. 3b) in each model. We selected two models that exhibited the most consistent expected BAF of SNPs (62.1 and $82.1 \%$ ) with that obtained by the WGS $(71.0 \%$; Fig. 3a). In these models, the chromosomal structure model of two mutant alleles without a wild-type allele showed the more consistent expected VAF of H3F3A K27M (64.1\%) with those obtained by ddPCR (68.0\%) than model of two mutant alleles with a wild-type allele did (Fig. 3c and Additional file 4: Figure S4). For case 12, WGS revealed two candidate models that exhibited one or two copy regions on $1 \mathrm{q}$ around which the $H 3 F 3 \mathrm{~A}$ gene was located in tumor cells (Fig. 3a). Based on the tumor content of the tumor specimen (90.6\%; Fig. 3b), we calculated the expected BAF of the common SNPs in each model and found that these two models exhibited consistent BAF (91.4 and 95.3\%) with that obtained by WGS (85.0\%; Fig. 3a). In these models, we calculated the expected VAF of H3F3A K27M (82.8 and 90.6\%) using the tumor content of the tumor specimen (90.6\%; Fig. 3b). Then, we selected the chromosomal structure model that exhibited the more consistent VAF of $H 3 F 3 A$ K27M (82.8\%) with those obtained by ddPCR (79.9\%). The most appropriate chromosomal structure model of this case was the partial loss of 1q containing the wildtype $H 3 F 3 A$ allele (Fig. $3 c$ and Additional file 5: Figure S5). In all these cases, we confirmed that the calculated tumor contents based on the most appropriate chromosomal structure model were consistent with those of the tumor specimen (Additional file 2: Figure S2, Additional file 3: Figure S3, Additional file 4: Figure S4 and Additional file 5: Figure S5). These data revealed that all four cases exhibited MASI of H3F3A K27M mutant with (cases 5,10, and 12) or without (case 15) loss of the wildtype allele (Fig. 3c). We also found consistent copy number variations on chromosome $1 \mathrm{p}$ and $1 \mathrm{q}$ in all MASI cases using the FISH assay (Fig. 3d), although FISH assay also revealed slight heterogeneous copy number variations on chromosome $1 \mathrm{p}$ and $1 \mathrm{q}$ in these cases.

\section{Aggressive phenotype in cases exhibiting MASI of $\mathrm{H} 3 \mathrm{~F} 3 \mathrm{~A}$ K27M}

During tumor formation, H3 K27M plays a pivotal role via the global reduction of H3K27me3 modification. Therefore, we investigated the expression level of $\mathrm{H} 3$ $\mathrm{K} 27 \mathrm{M}$ and H3K27me3 in MASI of H3F3A K27M cases with IHC. IHC revealed that the MASI cases $(n=4)$ exhibited a significantly higher expression level of $\mathrm{H} 3$ $\mathrm{K} 27 \mathrm{M}$ and lower level of $\mathrm{H} 3 \mathrm{~K} 27 \mathrm{me} 3$ than those in cases with lower VAF $(n=6)(P<0.05$ and $P<0.01$, respectively) (Fig. 4a-c, Table 1) together with comparable tumor cellularity between lower VAF cases and MASI cases (mean: 77.0 and $79.4 \%$, respectively). We validated these findings using Western blot analysis in the two available cases (Additional file 6: Figure S6). Additionally, the four MASI cases showed a significantly higher Ki-67 index than that in the lower VAF cases $(P<0.05)$ (Fig. 4d and e). These data revealed that the MASI of H3F3A K27M mutation was associated with promoted cell proliferation, which might be caused via the upregulation of H3 K27M expression. Next, we investigated the characteristic clinical features of the MASI cases. Compared with the lower VAF cases $(n=11)$, MASI cases $(n=4)$ did not exhibit characteristic clinical features such as age and tumor location; however, MASI cases exhibited a significantly poorer PFS ( $P=0.03$; Fig. $4 \mathrm{f})$ and OS $(P=0.01$; Fig. $4 \mathrm{~g})$ compared with the lower VAF cases. TP53 and ATRX gene mutations were previously reported to be the most frequently found gene mutations in diffuse midline glioma, H3 K27M-mutant cases [21]. In our case, we also found TP53 gene mutations $(n=3$; case 5, 12 and 14$)$ and ATRX gene mutations ( $n=3$; case 2, 7 and 12) however, these gene mutations were not significantly correlated with poorer prognosis and followed the same correlation even in the presence of MASI. These data revealed that the MASI of H3F3A $K 27 M$ was associated with the aggressive phenotype of diffuse midline glioma, H3 K27M-mutant.

\section{Discussion}

In the present study, we found that several diffuse midline glioma, H3 K27M-mutant exhibited higher VAF than $50 \%$ obtained by ddPCR assay. To the best of our 


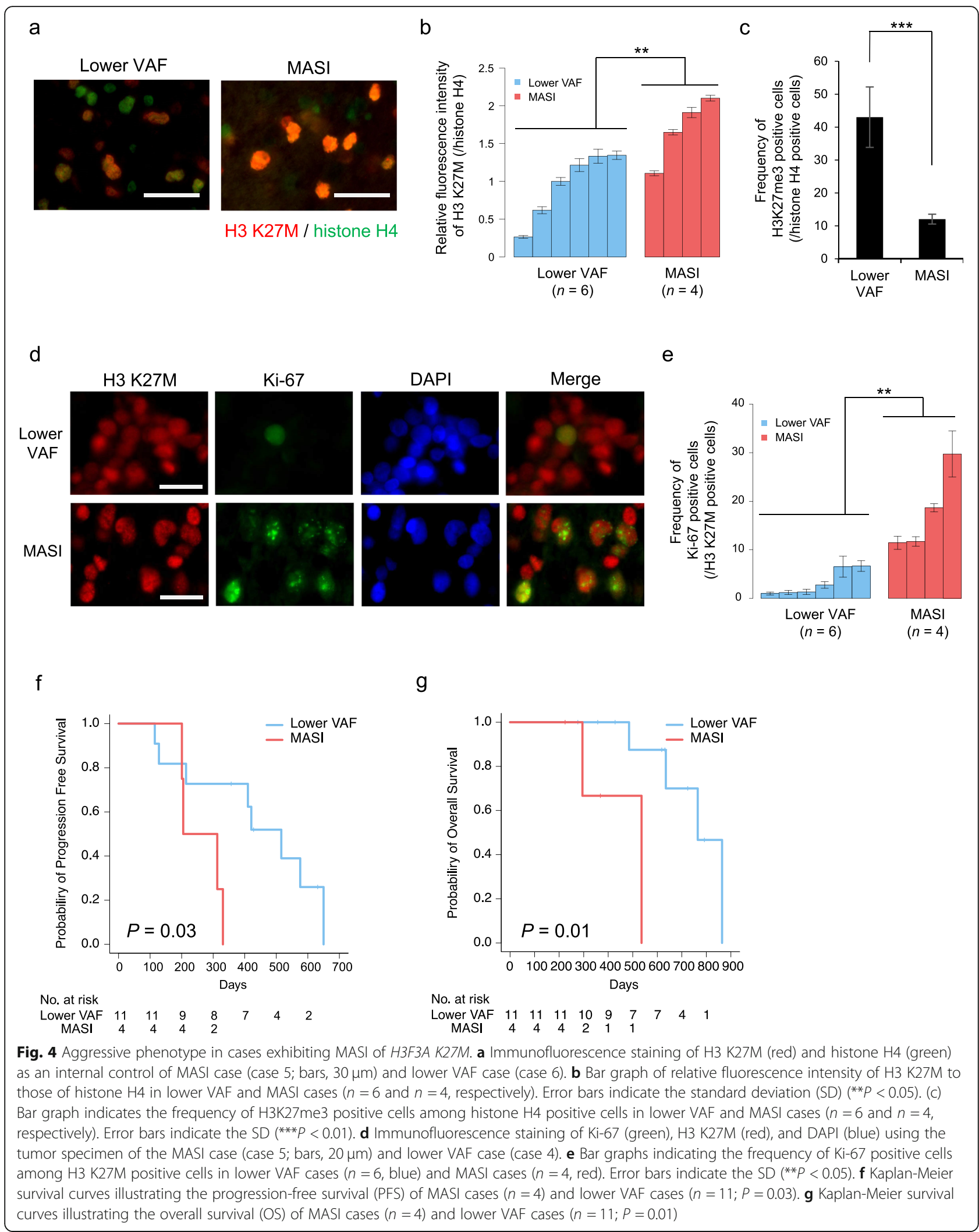


knowledge, our study is the first study to identify the MASI of $H 3 F 3 A K 27 M$ cases in these types of glioma by WGS analyses. MASI cases exhibited a significantly higher Ki-67 index in the tumor specimens and poorer survival than those of lower VAF cases; therefore, MASI was associated with an aggressive phenotype of this type of glioma. H3 K27M is known to inhibit the methyltransferase activity of PRC2 that catalyzes the trimethylation of $\mathrm{H} 3 \mathrm{~K} 27$, resulting in a reduction in the overall H3K27me3 level [22, 23]. Consistently, our study revealed that the MASI cases exhibited significantly lower H3K27me3 levels together with higher expression levels of H3 K27M compared with those in the lower VAF cases. In the human colorectal cancer cell lines, the MASI of the KRAS gene mutation has been reported to be sensitive to MEK inhibitors [12]. These data indicated that upregulated $\mathrm{H} 3 \mathrm{~K} 27 \mathrm{M}$ might be a potent treatment target for diffuse midline glioma, H3 K27M-mutant exhibiting MASI of $H 3 F 3 A$ K $27 M$.

There were previous studies where KRAS MASI elevated KRAS mRNA levels, increasing RAS activity [14, 24] and wild-type allele of KRAS has also been shown to play a pivotal role as a tumor suppressor [25]. Even in cases exhibiting a conventional heterozygous mutation of $H 3 F 3 A$ gene genetically, downregulation of wild-type $H 3 F 3 A$ via epigenetic dysregulation might contribute to the aggressive phenotype of this type of glioma, although further investigation is required.

MASI was considered to play a pivotal role in obtaining a selective growth advantage by increasing and tuning the dosage of mutations of oncogenic driver genes in cancer cells. The modest gain of the mutant allele or loss of the wild-type allele enhanced fitness and contributed to clonal outgrowth in most oncogenes [11]. These findings suggested that a subset of tumor cells exhibiting heterozygous mutation of $H 3 F 3 A K 27 M$ might acquire MASI of $H 3 F 3 A K 27 M$ during tumor formation of an aggressive subtype of diffuse midline glioma, H3 K27Mmutant. In the present study, we demonstrated that the MASI of H3F3A K27M mutation was associated with the aggressive phenotype via upregulation of H3 K27M together with downregulation of H3K27me3 in this type of glioma, resulting in a poor prognosis. Further analysis in a larger cohort may reinforce our conclusion because our total sample numbers were small $(n=15)$. Further investigation of the molecular mechanisms by which MASI of H3F3A K27M is associated with the aggressive phenotype of these cases might contribute to elucidating the tumor formation mechanism of an aggressive subset of diffuse midline glioma, H3 K27M-mutant.

\section{Conclusion}

DdPCR and WGS analyses revealed that the copy number gain of the mutant allele and/or loss of the wild-type allele of $H 3 F 3 A$ gene constituted MASI of $H 3 F 3 A$ K27M in diffuse midline glioma, H3 K27M-mutant. MASI of H3F3A K27M mutation was closely associated with an aggressive subtype of diffuse midline glioma, H3 K27Mmutant.

\section{Supplementary information}

Supplementary information accompanies this paper at https://doi.org/10. 1186/s40478-020-0882-4.

\begin{abstract}
Additional file 1: Figure S1. Copy numbers of whole chromosomes in four MASI cases. Total copy number of whole chromosomes obtained by WGS in four MASI cases (case 5, 10,12 and 15). Case 5, 10 and 15 were male ( $X$ : one and $Y$ : one). Case 12 was female ( $X$ : two).

Additional file 2: Figure S2. Flowchart indicating identification of the most appropriated chromosomal structure model in case 5. Total copy number of 1 q obtained by WGS ( $3 \leqq)$, tumor content in tumor specimen (64.2\%), BAF of SNPS obtained by WGS (91.0\%), and VAF of H3F3A K27M obtained by ddPCR (73.2\%) were used to reveal the most appropriate model of the $1 \mathrm{q}$ arm of tumor cells. The calculated tumor content with VAF of H3F3A K27M in the most appropriate model (64.6\%) was consistent with that of the tumor specimen (64.2\%).
\end{abstract}

Additional file 3: Figure S3. Flowchart indicating identification of the most appropriated chromosomal structure model in case 15. Total copy number of $1 \mathrm{q}$ obtained by WGS ( $3 \leqq)$, tumor content in tumor specimen (98.8\%), BAF of SNPs obtained by WGS (66.0\%), and VAF of H3F3A K27M obtained by ddPCR (65.9\%) were used to reveal the most appropriate model of $1 \mathrm{q}$ arm of tumor cells. The calculated tumor content with VAF of H3F3A K27M in the most appropriate model (98.3\%) was consistent with that of the tumor specimen (98.8\%).

Additional file 4: Figure S4. Flowchart indicating identification of the most appropriate chromosomal structure model in case 10. Total copy number of $1 q$ obtained by WGS $(2 \leqq)$, tumor content in tumor specimen (64.1\%), BAF of SNPs obtained by WGS (71.0\%), and VAF of H3F3A K27M obtained by ddPCR (68.0\%) were used to reveal the most appropriate model of $1 \mathrm{q}$ arm of tumor cells. The calculated tumor content with VAF of H3F3A K27M in the most appropriate model (68.0\%) was consistent with that of the tumor specimen (64.1\%).

Additional file 5: Figure S5. Flowchart indicating identification of the most appropriate chromosomal structure model in case 12. Total copy number of $1 \mathrm{q}$ obtained by WGS $(2 \geqq)$, tumor content in tumor specimen (90.6\%), BAF of SNPs obtained by WGS (85.0\%), and VAF of H3F3A K27M obtained by ddPCR (79.9\%) were used to reveal the most appropriate model of $1 \mathrm{q}$ arm of tumor cells. The calculated tumor content with VAF of H3F3A K27M in the most appropriate model (88.8\%) was consistent with that in of the tumor specimen (90.6\%).

Additional file 6: Figure S6. Protein expression levels of $\mathrm{H} 3 \mathrm{~K} 27 \mathrm{M}$ and H3K27me3. Protein expression level of H3 K27M (upper) and H3K27me3 (lower) in lower VAF case (case 6) and MASI case (case 10). H3F3A wildtype gliomas (IDH1 mutated) sample was used for a negative control (NC) for H3 K27M and a positive control (PC) for H3K27me3. Histone H3 protein expression level was used as an internal control.

\section{Abbreviations}

BAF: B Allele Frequency; ddPCR: Droplet Digital Polymerase Chain Reaction; DIPG: Diffuse Intrinsic Pontine Glioma; FFPE: Formalin-Fixed ParaffinEmbedded; FISH: Fluorescence in situ hybridization;

IHC: Immunohistochemistry; MASI: Mutant Allele Specific Imbalance; OS: Overall Survival; PFS: Progression-Free Survival; SD: Standard Deviation; SNPS: Single Nucleotide Polymorphisms; VAF: Variant Allele Frequency; WGS: Whole-Genome Sequencing

Acknowledgements Not applicable. 


\section{Authors' contributions}

Conception and design: F.O., Y.O., A.N.; development of methodology: S.M., F.O., Y.O., K.A., H.K., N.Y., S.S.; acquisition of data: S.M., F.O., Y.O., K.A., K.M., K.T. H.K., N.Y., S.S., J.Y., K.T., M.H., A.K., H.S., Y.K., S.Y., S.Y., H.T., K.S., Y.K.; analysis and interpretation of data: S.M., F.O., Y.O., K.A., A.N.; writing of the manuscript: S.M., F.O., Y.O., K.A., A.N. All authors read and approved the final manuscript.

\section{Funding}

This study was performed as a research program of the Grant-in-Aid for Scientific Research, the Japan Society for the Promotion of Science (17928985, A. Natsume; 19 K09478, F. Ohka).

\section{Availability of data and materials}

The datasets generated during the current study are available in the National Bioscience Database Center (NBDC) repository (https://biosciencedbc.jp/en/).

\section{Ethics approval and consent to participate}

The present study was approved by the institutional review board at Nagoya University Hospital (approval number: 2012-0067) and it complied with all provisions of the World Medical Association Declaration of Helsinki. Tumor samples of sequential diffuse midline glioma, H3 K27M-mutant cases that had undergone biopsy from February 2014 to March 2019 were collected intra-operatively upon receiving informed consent from the patients.

\section{Consent for publication}

Not applicable.

\section{Competing interests}

The authors declare that they have no competing interests.

\section{Author details}

'Department of Neurosurgery, Nagoya University Graduate School of Medicine, 65 Tsurumai-cho, Showa-ku, Nagoya 466-8550, Japan. ${ }^{2}$ Medical Genomics Center, Nagoya University Hospital, 65 Tsurumai-cho, Showa-ku, Nagoya 466-8550, Japan. ${ }^{3}$ Department of Pathology, St. Marianna University School of Medicine Yokohama City Seibu Hospital, 1197-1 Yasashi-cho, Asahi-ku, Yokohama 241-0811, Japan. ${ }^{4}$ Molecular Pathology and Genetics Division, Kanagawa Cancer Center Research Institute, 2-3-2 Nakao, Asahi-ku, Yokohama 241-8515, Japan. ${ }^{5}$ Department of Neurosurgery, Division of Clinical Neuroscience, Faculty of Medicine, University of Miyazaki, 5200, Kiyotake-cho, Kihara, Miyazaki 889-1601, Japan. ${ }^{6}$ Division of Cancer Biology, Nagoya University Graduate School of Medicine, Nagoya, Japan, 65 Tsurumai-cho, Showa-ku, Nagoya 466-8550, Japan.

Received: 3 December 2019 Accepted: 18 January 2020 Published online: 05 February 2020

\section{References}

1. Buczkowicz P, Hoeman C, Rakopoulos P, Pajovic S, Letourneau L, Dzamba M, Morrison A, Lewis P, Bouffet E, Bartels U et al (2014) Genomic analysis of diffuse intrinsic pontine gliomas identifies three molecular subgroups and recurrent activating ACVR1 mutations. Nat Genet 46:451-456. https://doi. org/10.1038/ng.2936

2. Fontebasso AM, Papillon-Cavanagh S, Schwartzentruber J, Nikbakht $H$, Gerges N, Fiset PO, Bechet D, Faury D, De Jay N, Ramkissoon LA et al (2014) Recurrent somatic mutations in ACVR1 in pediatric midline high-grade astrocytoma. Nat Genet 46:462-466. https://doi.org/10.1038/ng.2950

3. Schwartzentruber J, Korshunov A, Liu XY, Jones DT, Pfaff E, Jacob K, Sturm D, Fontebasso AM, Quang DA, Tonjes M et al (2012) Driver mutations in histone $\mathrm{H} 3.3$ and chromatin remodelling genes in paediatric glioblastoma. Nature 482:226-231. https://doi.org/10.1038/nature10833

4. Taylor KR, Mackay A, Truffaux N, Butterfield Y, Morozova O, Philippe C, Castel D, Grasso CS, Vinci M, Carvalho D et al (2014) Recurrent activating ACVR1 mutations in diffuse intrinsic pontine glioma. Nat Genet 46:457-461. https:// doi.org/10.1038/ng.2925

5. Wu G, Broniscer A, McEachron TA, Lu C, Paugh BS, Becksfort J, Qu C, Ding L, Huether R, Parker M et al (2012) Somatic histone H3 alterations in pediatric diffuse intrinsic pontine gliomas and non-brainstem glioblastomas. Nat Genet 44:251-253. https://doi.org/10.1038/ng.1102

6. Wu G, Diaz AK, Paugh BS, Rankin SL, Ju B, Li Y, Zhu X, Qu C, Chen X, Zhang $J$ et al (2014) The genomic landscape of diffuse intrinsic pontine glioma and pediatric non-brainstem high-grade glioma. Nat Genet 46:444-450. https://doi.org/10.1038/ng.2938

7. Louis DN, Perry A, Reifenberger G, von Deimling A, Figarella-Branger D, Cavenee WK, Ohgaki H, Wiestler OD, Kleihues P, Ellison DW (2016) The 2016 World Health Organization classification of tumors of the central nervous system: a summary. Acta Neuropathol 131:803-820. https://doi.org/10.1007/ s00401-016-1545-1

8. Harutyunyan AS, Krug B, Chen H, Papillon-Cavanagh S, Zeinieh M, De Jay N, Deshmukh S, Chen CCL, Belle J, Mikael LG et al (2019) H3K27M induces defective chromatin spread of PRC2-mediated repressive H3K27me2/me3 and is essential for glioma tumorigenesis. Nat Commun 10:1262. https://doi. org/10.1038/s41467-019-09140-x

9. Venneti S, Garimella MT, Sullivan LM, Martinez D, Huse JT, Heguy A, Santi M, Thompson CB, Judkins AR (2013) Evaluation of histone 3 lysine 27 trimethylation ( $\mathrm{H} 3 \mathrm{~K} 27 \mathrm{me} 3)$ and enhancer of zest $2(\mathrm{EZH} 2)$ in pediatric glial and glioneuronal tumors shows decreased H3K27me3 in H3F3A K27M mutant glioblastomas. Brain Pathol 23:558-564. https://doi.org/10.1111/bpa. 12042

10. Venneti S, Santi M, Felicella MM, Yarilin D, Phillips JJ, Sullivan LM, Martinez D, Perry A, Lewis PW, Thompson CB et al (2014) A sensitive and specific histopathologic prognostic marker for H3F3A K27M mutant pediatric glioblastomas. Acta Neuropathol 128:743-753. https://doi.org/10.1007/ s00401-014-1338-3

11. Bielski CM, Donoghue MTA, Gadiya M, Hanrahan AJ, Won HH, Chang MT, Jonsson P, Penson AV, Gorelick A, Harris C et al (2018) Widespread selection for oncogenic mutant allele imbalance in Cancer. Cancer Cell 34(852-862): e854. https://doi.org/10.1016/j.ccell.2018.10.003

12. Burgess MR, Hwang E, Mroue R, Bielski CM, Wandler AM, Huang BJ, Firestone AJ, Young A, Lacap JA, Crocker L et al (2017) KRAS allelic imbalance enhances fitness and modulates MAP kinase dependence in Cancer. Cell 168(817-829):e815. https://doi.org/10.1016/j.cell.2017.01.020

13. Malapelle U, Sgariglia R, De Stefano A, Bellevicine C, Vigliar E, de Biase D, Sepe R, Pallante P, Carlomagno C, Tallini G et al (2015) KRAS mutant allelespecific imbalance (MASI) assessment in routine samples of patients with metastatic colorectal cancer. J Clin Pathol 68:265-269. https://doi.org/10. 1136/jclinpath-2014-202761

14. Soh J, Okumura N, Lockwood WW, Yamamoto H, Shigematsu H, Zhang W, Chari R, Shames DS, Tang X, MacAulay C et al (2009) Oncogene mutations, copy number gains and mutant allele specific imbalance (MASI) frequently occur together in tumor cells. PLoS One 4:e7464. https://doi.org/10.1371/ journal.pone.0007464

15. Valtorta E, Misale S, Sartore-Bianchi A, Nagtegaal ID, Paraf F, Lauricella C, Dimartino V, Hobor S, Jacobs B, Ercolani C et al (2013) KRAS gene amplification in colorectal cancer and impact on response to EGFRtargeted therapy. Int J Cancer 133:1259-1265. https://doi.org/10.1002/ijc. 28106

16. Yu CC, Qiu W, Juang CS, Mansukhani MM, Halmos B, Su GH (2017) Mutant allele specific imbalance in oncogenes with copy number alterations: occurrence, mechanisms, and potential clinical implications. Cancer Lett 384:86-93. https://doi.org/10.1016/j.canlet.2016.10.013

17. Krasinskas AM, Moser AJ, Saka B, Adsay NV, Chiosea SI (2013) KRAS mutant allele-specific imbalance is associated with worse prognosis in pancreatic cancer and progression to undifferentiated carcinoma of the pancreas. Mod Pathol 26:1346-1354. https://doi.org/10.1038/modpathol.2013.71

18. Seshan VE, Olshen AB (2010) DNAcopy: a package for analyzing DNA copy data

19. Lu C, Ward PS, Kapoor GS, Rohle D, Turcan S, Abdel-Wahab O, Edwards CR, Khanin R, Figueroa ME, Melnick A et al (2012) IDH mutation impairs histone demethylation and results in a block to cell differentiation. Nature 483:474478. https://doi.org/10.1038/nature10860

20. Mackay A, Burford A, Carvalho D, Izquierdo E, Fazal-Salom J, Taylor KR, Bjerke L, Clarke M, Vinci M, Nandhabalan M et al (2017) Integrated molecular meta-analysis of 1,000 pediatric high-grade and diffuse intrinsic Pontine Glioma. Cancer Cell 32(520-537):e525. https://doi.org/10.1016/j.ccell. 2017.08.017

21. Nikbakht H, Panditharatna E, Mikael LG, Li R, Gayden T, Osmond M, Ho CY, Kambhampati M, Hwang El, Faury D et al (2016) Spatial and temporal homogeneity of driver mutations in diffuse intrinsic pontine glioma. Nat Commun 7:11185. https://doi.org/10.1038/ncomms11185

22. Lewis PW, Muller MM, Koletsky MS, Cordero F, Lin S, Banaszynski LA, Garcia BA, Muir TW, Becher OJ, Allis CD (2013) Inhibition of PRC2 activity by a gain- 
of-function H3 mutation found in pediatric glioblastoma. Science 340:857861. https://doi.org/10.1126/science.1232245

23. Mohammad F, Weissmann S, Leblanc B, Pandey DP, Højfeldt JW, Comet I, Zheng C, Johansen JV, Rapin N, Porse BT et al (2017) EZH2 is a potential therapeutic target for H3K27M-mutant pediatric gliomas. Nat Med 23:483492. https://doi.org/10.1038/nm.4293

24. Aguirre AJ, Bardeesy N, Sinha M, Lopez L, Tuveson DA, Horner J, Redston MS, DePinho RA (2003) Activated Kras and Ink4a/Arf deficiency cooperate to produce metastatic pancreatic ductal adenocarcinoma. Genes Dev 17:31123126. https://doi.org/10.1101/gad.1158703

25. Zhang Z, Wang Y, Vikis HG, Johnson L, Liu G, Li J, Anderson MW, Sills RC, Hong HL, Devereux TR et al (2001) Wildtype Kras2 can inhibit lung carcinogenesis in mice. Nat Genet 29:25-33. https://doi.org/10.1038/ng721

\section{Publisher's Note}

Springer Nature remains neutral with regard to jurisdictional claims in published maps and institutional affiliations.

Ready to submit your research? Choose BMC and benefit from:

- fast, convenient online submission

- thorough peer review by experienced researchers in your field

- rapid publication on acceptance

- support for research data, including large and complex data types

- gold Open Access which fosters wider collaboration and increased citations

- maximum visibility for your research: over $100 \mathrm{M}$ website views per year

At BMC, research is always in progress.

Learn more biomedcentral.com/submissions 